\title{
Crescimento e distribuição de raízes de soja em diferentes densidades de plantas
}

\author{
Growth and distribution of soybean roots at different plant densities
}

\author{
Alvadi Antonio Balbinot Junior ${ }^{1}$, Henrique Debiasi ${ }^{1}$, Julio Cezar Franchini ${ }^{1}$, João Pedro Carlos \\ Prieto $^{2}$, Moacir Tuzzin de Moraes ${ }^{3}$, Flávia Werner ${ }^{4 *}$ \& André Sampaio Ferreira ${ }^{4}$ \\ ${ }^{1}$ Empresa Brasileira de Pesquisa Agropecuária, Londrina, PR, Brasil. \\ ${ }^{2}$ Coamo Agroindustrial Cooperativa, Borrazópolis, PR, Brasil. \\ ${ }^{3}$ Universidade de São Paulo, Centro de Energia Nuclear na Agricultura, Seção de Isótopos Estáveis, SP, Brasil. \\ ${ }^{4}$ Universidade Estadual de Londrina, Londrina, PR, Brasil. *Autor para correspondência: fla_werner@hotmail.com.
}

Submissão: 28/06/2016 / Aceite: 30/10/2017

\begin{abstract}
RESUMO
A cultura da soja apresenta alta plasticidade fenotípica da parte aérea, alterando a sua morfologia em função da densidade de plantas. No entanto, os seus efeitos no crescimento e na distribuição de raízes no perfil do solo ainda não estão elucidados. O objetivo do presente trabalho foi avaliar o efeito de três densidades de plantas de soja sobre variáveis relacionadas ao crescimento de raízes, em diferentes camadas de solo e posições (linha e entrelinhas). Foram conduzidos dois experimentos na safra 2014/2015 em Londrina, PR, um com a cultivar BRS 359 RR e outro com a cultivar BMX Potência RR. O delineamento experimental foi de blocos casualizados, com três repetições e parcelas subsubdivididas. Nas parcelas principais, foram alocadas três densidades de semeadura (150, 300 e 450 mil sementes ha $\left.{ }^{-1}\right)$; nas subparcelas, cinco camadas de solo (0-10, 10-20, 20-30, 30-40 e $\left.40-50 \mathrm{~cm}\right)$; e nas subsubparcelas, três posições (linha, entrelinha à esquerda e entrelinha à direita). $\mathrm{O}$ aumento da densidade de plantas provoca a formação de raízes mais finas, com tendência de haver maior ocupação das entrelinhas e das camadas subsuperficiais do solo, mas a magnitude dessa resposta é dependente da cultivar.
\end{abstract}

PALAVRAS-CHAVE: Glycine max L., densidade de massa radicular, área superficial radicular, densidade do comprimento radicular, diâmetro de raízes.

\begin{abstract}
The soybean plant has long been known to have a high phenotypic plasticity, altering shoot morphological traits in response to variations on plant density. However, plant density effects on soybean root growth and distribution in the soil profile are not elucidated. This paper aimed at evaluating the effects of three plant densities on soybean root growth at different soil layers and horizontal positions relative to the crop planting row. The study comprised two field experiments carried out during 2014/2015 growing season in Londrina, PR, Southern Brazil, one with the soybean cultivar BRS 359 RR, and other with the cultivar BMX Potência RR. Both experiments were laid out in a randomized complete block design and split-split-plot arrangement, with three replications. In the main plots, three soybean seeding rates (150; 300; and 450 thousand seeds ha $\left.^{-1}\right)$ were distributed; in the subplots, five soil layers $(0-10 ; 10-20 ; 20-30 ; 30-40$ and 40-50 $\mathrm{cm}$ depth); and, in the sub-subplots, three horizontal sampling positions (row; interrow on the left; and interrow on the right). Higher soybean plant densities led to thinner roots, with greater growth towards the crop interrows and deeper soil layers, but the cultivars can affect this behavior.
\end{abstract}

KEYWORDS: Glycine max L., root dry matter density, root surface area, root length density, root diameter.

\section{INTRODUÇÃO}

Na última década, a cultura da soja apresentou aumentos expressivos de área e de produtividade no Brasil. O ajuste nas práticas de manejo, considerando as características genéticas e dos ambientes de produção, colaborou para o fortalecimento da cultura no país. Entre as práticas de manejo, a densidade de plantas altera a competição intraespecífica e, consequentemente, a quantidade de recursos do ambiente, água, luz e nutrientes, disponíveis para cada indivíduo (GASPAR \& CONLEY 2015). 
A competição intraespecífica pode influenciar a velocidade de fechamento das entrelinhas (COX \& CHERNEY 2011), a produção de fitomassa aérea (PROCÓPIO et al. 2013), a arquitetura das plantas (BALBINOT JUNIOR et al. 2015a), a severidade de doenças (HANNA et al. 2008), o acamamento (BALBINOT JUNIOR et al. 2015b), os componentes de rendimento (COX \& CHERNEY 2011) e a produtividade de grãos das culturas (BALBINOT JUNIOR et al. 2015a). Em geral, baixas densidades (inferiores a 200 mil plantas ha $^{-1}$ ) causam a formação de plantas robustas, com vigorosa ramificação lateral, mas baixa estatura.

Vários trabalhos têm demonstrado que variações na densidade de plantas de soja influenciam a arquitetura das plantas e o número de vagens por planta, mas, há reduzida variação na produtividade de grãos por área (PROCÓPIO et al. 2013, BALBINOT JUNIOR et al. 2015a,b). Esse resultado é função da alta plasticidade fenotípica da cultura, a qual consiste na capacidade da planta em alterar a sua morfologia e os componentes do rendimento a fim de adequá-los à condição imposta pelo arranjo de plantas, permitindo a manutenção da produtividade em ampla faixa de densidade (COX \& CHERNEY 2011). Nesse contexto, as principais variações morfofisiológicas que ocorrem na parte aérea das plantas em função da densidade já estão relativamente bem elucidadas, mas as alterações no crescimento e na distribuição de raízes no perfil do solo em relação à densidade ainda permanecem pouco compreendidas.

O estudo da plasticidade fenotípica de raízes quanto ao ambiente de cultivo tem recebido maior atenção na última década em razão do grande impacto que essa variável pode apresentar sobre a produção vegetal (POSTMA et al. 2014). Nesse sentido, a avaliação do sistema radicular das culturas pode aprimorar o entendimento da interação solo-planta-atmosfera, sendo a base para a adoção de práticas de manejo que visem incrementar a exploração do solo pelas raízes em busca de água e nutrientes (BORDIN et al. 2008, FAN et al. 2016), o que pode resultar em incrementos de produtividade ou da estabilidade produtiva (WHITE \& KIRKEGAARD 2010). Adicionalmente, a caracterização da resposta das raízes da soja a práticas de manejo, sob diferentes condições de cultivo, é essencial para o aprimoramento de modelos que quantifiquem a dinâmica do crescimento radicular da cultura para estudos de fluxo de água no solo e absorção de água e de nutrientes.

Nesse contexto, o objetivo do presente trabalho foi avaliar o efeito de três densidades de plantas de soja sobre variáveis relacionadas ao crescimento de raízes, em diferentes camadas de solo e posições em relação à linha de semeadura.

\section{MATERIAL E MÉTODOS}

O trabalho foi desenvolvido na Fazenda Experimental da Embrapa Soja, em Londrina, PR (latitude $23^{\circ} 11^{\prime} \mathrm{S}$, longitude $51^{\circ} 11^{\prime} \mathrm{W}$ e altitude de $620 \mathrm{~m}$ ), na safra $2014 / 2015$. O solo foi identificado como Latossolo Vermelho Distroférrico, apresentando os seguintes atributos, na camada de 0 a $20 \mathrm{~cm}$, antes da

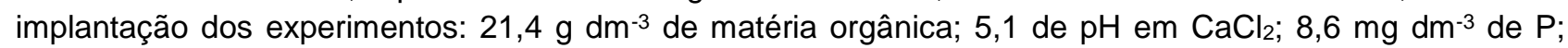
$0,55 \mathrm{cmol}_{\mathrm{c}} \mathrm{dm}^{-3}$ de $\mathrm{K} ; 3,7 \mathrm{cmol}_{\mathrm{c}} \mathrm{dm}^{-3}$ de Ca; $1,4 \mathrm{cmol}_{\mathrm{c}} \mathrm{dm}^{-3} \mathrm{de} \mathrm{Mg}$; e $58 \%$ de saturação da CTC por bases. O solo não apresentava restrições físicas ou químicas relevantes que pudessem reduzir o crescimento das raízes de soja. A resistência à penetração na camada de solo de $0-50 \mathrm{~cm}$, com umidade na capacidade de campo, variou de 1,5 a 2,5 MPa.

A cobertura vegetal presente na área experimental, formada por aveia preta, foi manejada mecanicamente aos 11 dias antes da semeadura da soja utilizando-se um triturador de restos culturais (triton). Nove dias após essa operação, a vegetação remanescente foi dessecada quimicamente com glyphosate $\left(1.080 \mathrm{~g} \mathrm{ha}^{-1}\right)$ e carfentrazone-ethyl $\left(30 \mathrm{~g} \mathrm{ha}^{-1}\right)$. A semeadura da soja foi realizada no dia $11 \mathrm{de}$ novembro de 2014 em sistema plantio direto. As sementes foram tratadas com Vitavax-Thiran 200 SC $^{\circledR}(150$ $\mathrm{mL} 50 \mathrm{~kg}^{-1}$ de sementes) e inoculante líquido Gelfix $5^{\circledR}\left(100 \mathrm{~mL} 50 \mathrm{~kg}^{-1}\right.$ de sementes). A adubação de base constou da aplicação de $122 \mathrm{~kg} \mathrm{ha}^{-1}$ de superfosfato triplo e de $250 \mathrm{~kg} \mathrm{ha}^{-1}$ de cloreto de potássio, aplicados a lanço 10 dias antes da semeadura. Os dados de precipitação pluvial e de temperaturas máxima, mínima e média do ar durante o período de condução dos experimentos estão apresentados na Figura 1.

Foram conduzidos dois experimentos, um com a cultivar BRS 359 RR e outro com a cultivar BMX Potência RR, ambas de crescimento indeterminado e grupo de maturidade relativa de 6.0 e 6.7, respectivamente. Essas cultivares, com ciclos distintos, foram selecionadas por apresentarem alto potencial de rendimento, arquitetura moderna de plantas e baixa propensão ao acamamento. $O$ delineamento experimental foi de blocos completos casualizados, em esquema de parcelas subsubdivididas, com três repetições. Nas parcelas, foram alocadas três densidades de semeadura $(150,300$ e 450 mil sementes ha-1), com espaçamento entrelinhas de $50 \mathrm{~cm}$. As densidades finais tiveram variação de $\pm 10 \%$ no valor pretendido. Nas subparcelas, foram avaliadas cinco camadas de solo (0-10, 10-20, 20-30, 30-40 e 
40-50 cm) e, nas subsubparcelas, três posições horizontais (linha, entrelinha à esquerda e entrelinha à direita). As parcelas apresentaram $50 \mathrm{~m}^{2}(5 \times 10 \mathrm{~m})$ e área útil de $24 \mathrm{~m}^{2}(3 \times 8 \mathrm{~m})$.

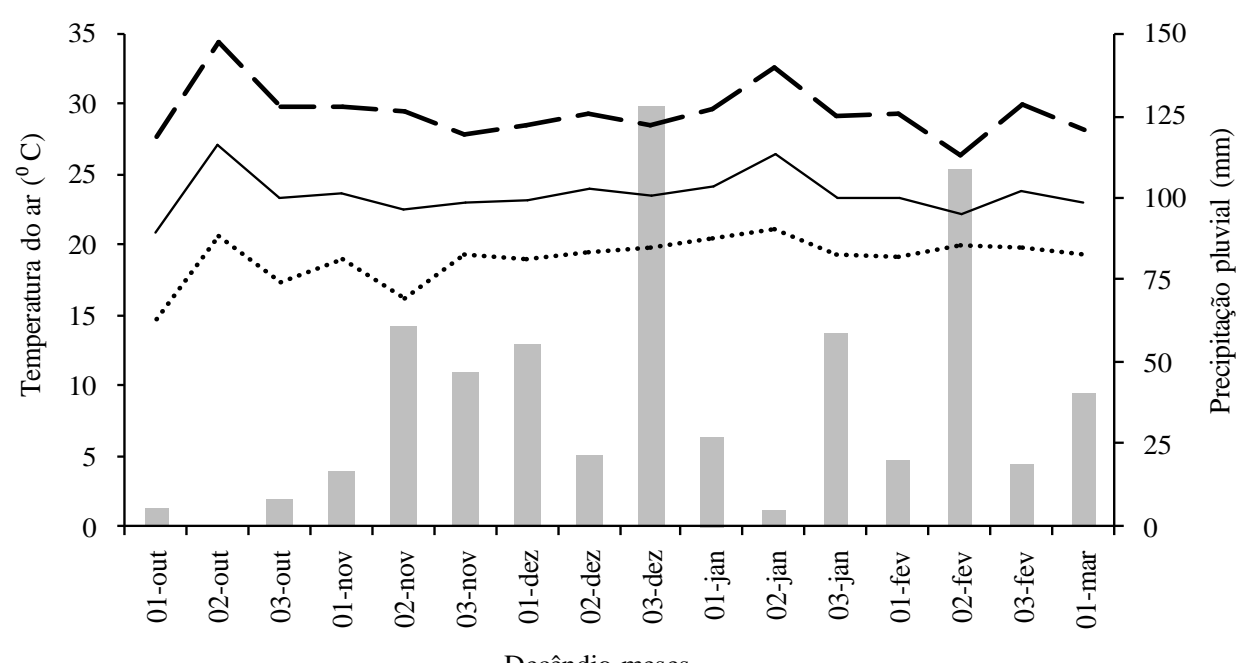

Decêndio-meses

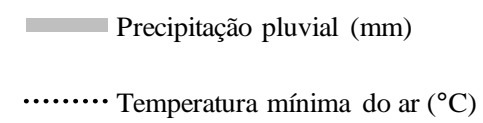

$$
\begin{aligned}
& \text { — Temperatura média do ar }\left({ }^{\circ} \mathrm{C}\right) \\
& \text { — — Temperatura máxima do } \operatorname{ar}\left({ }^{\circ} \mathrm{C}\right)
\end{aligned}
$$

Figura 1. Precipitação pluvial e temperaturas máxima, mínima e média do ar durante o ciclo de desenvolvimento da cultura da soja na safra 2014/2015. Londrina, PR.

Figure 1. Rainfall and maximum, minimum and average air temperature during the soybean crop development cycle in 2014/2015 growing season. Londrina, PR.

O sistema radicular da soja foi avaliado no início do enchimento de grãos (estádio R5.1), por meio da coleta de monólitos de solo com $17 \mathrm{~cm}$ de largura, $7 \mathrm{~cm}$ de espessura e $10 \mathrm{~cm}$ de profundidade, em trincheiras abertas perpendicularmente a uma linha de soja presente na área útil das parcelas. Em cada camada, o centro de um dos monólitos coincidiu com a linha de semeadura, sendo os outros dois posicionados imediatamente à esquerda (face oeste) e à direita (face leste) na entrelinha da cultura. Assim, a amostragem estendeu-se do centro de uma entrelinha até o centro da entrelinha mais próxima.

As raízes foram separadas do solo por meio de lavagem com água e com auxílio de peneiras de 0,5 $\mathrm{mm}$ de malha. Amostras representativas das raízes foram digitalizadas usando scanner óptico, com resolução de $200 \mathrm{dpi}$. Em seguida, as raízes foram secas em estufa com circulação forçada de ar ( $\pm 60{ }^{\circ} \mathrm{C}$, $48 \mathrm{~h}$ ), obtendo, assim, a massa seca das raízes digitalizadas e não digitalizadas de cada amostra. A partir das imagens digitalizadas, foram determinados o comprimento, a área superficial e o diâmetro radicular de cada amostra com o auxílio do programa Safira®, versão 2.0.

Tendo em vista a grande variação do diâmetro entre e dentro de uma mesma fibra radicular, o mesmo foi calculado como média ponderada em função do comprimento radicular equivalente a cada classe de diâmetro. A partir da massa seca total (digitalizada + não digitalizada) e do volume de cada monólito (1.170 $\left.\mathrm{cm}^{3}\right)$, obteve-se a densidade de massa radicular $\left(\mathrm{kg} \mathrm{m}^{-3}\right)$. Os dados obtidos a partir da análise das imagens foram relacionados à massa seca total da respectiva amostra e ao volume dos monólitos para quantificar a área superficial radicular $\left(\mathrm{m}^{2} \mathrm{~m}^{-3}\right)$ e a densidade do comprimento radicular $\left(\mathrm{cm} \mathrm{cm}^{-3}\right)$.

Os dados foram submetidos à análise de variância e teste $\mathrm{F}$, sendo as médias comparadas pelo teste Tukey $(p \leq 0,05)$. Em função da falta de homogeneidade, os dados de massa seca, área e comprimento de raízes foram transformados em $(x+1)^{1 / 2}$. As análises foram executadas por meio do programa computacional Sistema para Análise de Variância - SISVAR 5.6.

\section{RESULTADOS E DISCUSSÃO}

Na cultivar BRS 359 RR, houve efeito da interação tripla (densidade de plantas, posição de amostragem e camadas do solo) para todas as variáveis (densidade da massa, área superficial, densidade do comprimento e diâmetro médio radicular). Entretanto, na cultivar BMX Potência RR, ocorreram apenas interações duplas entre posição de amostragem e camadas avaliadas. 
A densidade de plantas na cultivar BRS 359 RR afetou a massa de raízes somente na camada superficial do solo e na linha de semeadura (Figura 2). Assim, a maior densidade de massa de raízes na linha de semeadura foi observada com uso da menor densidade de plantas. Provavelmente isso ocorreu em função da menor competição intraespecífica, favorecendo o crescimento das raízes na linha de semeadura e na camada superficial do solo, corroborando os resultados obtidos por KASPERBAUER (1987). Houve variação da massa seca de raízes entre camadas somente na linha de semeadura. Nessa posição, conforme esperado, a camada superficial apresentou maior massa em relação às camadas subsuperficiais. Nas entrelinhas, a massa de raízes não diferiu entre as camadas avaliadas, indicando que houve uma distribuição uniforme da massa radicular no perfil do solo. Nas densidades de 300 e 450 mil plantas ha-1 e na camada superficial do solo, houve maior crescimento de raízes na linha em relação às entrelinhas. No entanto, na menor densidade avaliada e na camada superficial, a massa de raízes na linha foi superior somente à entrelinha à direita.

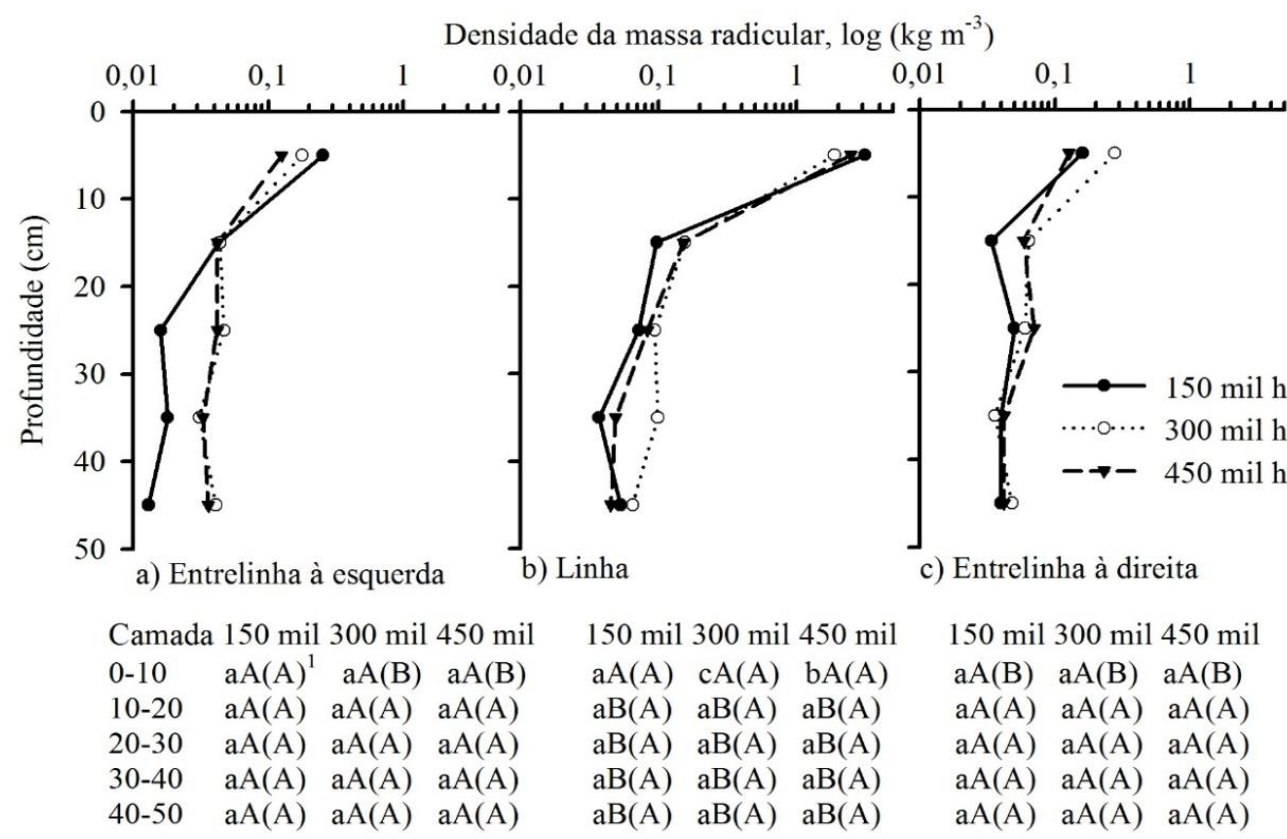

Figura 2. Densidade de massa radicular de soja (eixo x em escala log), cultivar BRS 359 RR, em diferentes camadas de solo, posições de amostragem e densidades de plantas. Londrina, PR, safra $2014 / 2015$.

${ }^{1}$ Letras minúsculas comparam as médias de densidade de plantas dentro de cada posição e camada de solo. Letras maiúsculas comparam as médias de camadas de solo dentro de cada posição e densidade de plantas. Letras entre parênteses comparam as médias de posições de amostragem dentro de cada densidade de plantas e camadas de solo (Tukey p $<0,05$ ). CV densidade $=6,7 \%$; CV camada de solo $=5,9 \%$ e CV da posição $=4,8 \%$. Dados transformados em $(x+1)^{1 / 2}$.

Figure 2. Soybean root dry matter density ( $x$ axis in log scale), cultivar BRS $359 R R$, in different soil layers, sampling positions and plant densities. Londrina, PR, 2014/2015 growing season.

${ }_{1}^{1}$ Lowercase letters compare averages of plant densities inside each position and soil layer. Uppercase letters compare averages of soil layers inside each position and plant densities. Letters in parentheses compare averages of sampling positions inside each plant densities and soil layers (Tukey $p<0.05$ ). CV density $=6.7 \%$; $\mathrm{CV}$ soil layer $=5.9 \%$ and $\mathrm{CV}$ position $=4.8 \%$. Transformed data $(x+1)^{1 / 2}$.

Na cultivar BMX Potência RR, a densidade de plantas não influenciou a massa de raízes (Figura 3a). Entretanto, houve efeito significativo de camadas e posição horizontal (Figura 3b), sendo os resultados similares aos obtidos para a cultivar BRS 359 RR (Figura 2). Assim, houve diferença entre camadas somente na linha de semeadura, na qual a maior densidade de massa seca de raízes da cultivar BMX Potência RR foi observada na camada de $0-10 \mathrm{~cm}$. Nessa mesma cultivar, as posições diferiram quanto à massa de raízes somente na camada superficial, com os maiores valores ocorrendo na linha, comparativamente às entrelinhas.

$\mathrm{Na}$ cultivar BRS $359 \mathrm{RR}$, houve efeito da interação tripla para a área de raízes (Figura 4). Na entrelinha à esquerda, nas camadas de 20-30 e 40-50 cm, contatou-se menor área de raízes na densidade de 150 mil plantas ha-1 em relação às maiores densidades. Na linha de semeadura, nas camadas de 30-40 e 40-50 cm, também se verificou menor área radicular na menor densidade. Contudo, na entrelinha à direita, a área radicular não foi influenciada pelas densidades. 
Densidade da massa radicular, $\log \left(\mathrm{kg} \mathrm{m}^{-3}\right)$

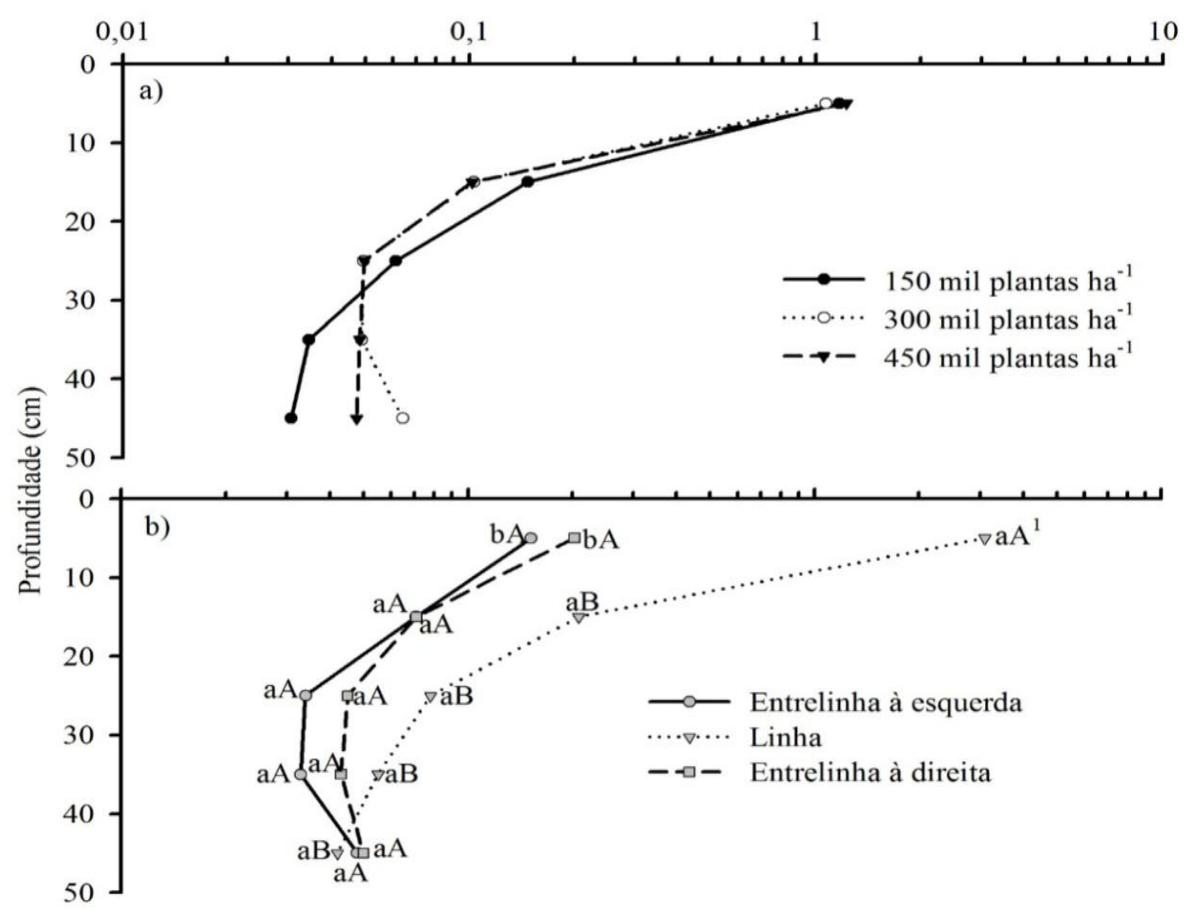

Figura 3. Densidade de massa radicular de soja (eixo $x$ em escala log), cultivar BMX Potência RR, em diferentes camadas de solo em função da densidade de plantas (a) e posições de amostragem (b). Londrina, PR, safra 2014/2015.

'Letras minúsculas comparam as médias de posição dentro da camada de solo. Letras maiúsculas comparam as médias de camadas de solo dentro de cada posição (Tukey $p<0,05)$. CV camada do solo = $7,6 \%$ e CV da posição $=9,1 \%$. Dados transformados em $(x+1)^{1 / 2}$.

Figure 3. Soybean root dry matter density ( $x$ axis in log scale), cultivar BMX Potência RR, in different soil layers due plant densities (a) and sampling position (b). Londrina, PR, 2014/2015 growing season. ${ }^{1}$ Lowercase letters compare averages of position inside the soil layer. Uppercase letters compare averages of soil layers in each position (Tukey $p<0.05$ ). $\mathrm{CV}$ soil layers $=7.6 \%$ and $\mathrm{CV}$ position $=9.1 \%$. Transformed data $(x+1)^{1 / 2}$.

Nas três posições avaliadas, na densidade de 150 mil plantas ha-1 $\pm 10 \%$, houve maior área de raízes na camada superficial em relação às demais. As diferenças entre camadas nas densidades maiores foram menos intensas, evidenciando melhor distribuição das raízes em profundidade. Houve maior área de raízes na linha do que nas entrelinhas na menor densidade na camada superficial, demonstrando que parte do volume do solo foi menos explorada pelo sistema radicular. Na densidade de 300 mil plantas ha-1 $\pm 10 \%$, não houve variação de área radicular entre as posições nas cinco camadas amostradas. $\mathrm{Na}$ maior densidade, observou-se menor área de raízes na linha e na entrelinha à direita em relação à entrelinha esquerda nas camadas de $20-30$ e $40-50 \mathrm{~cm}$.

A densidade do comprimento radicular foi alterada em função da densidade de plantas na cultivar BRS 359 RR (Figura 5). De maneira similar ao observado para a área superficial radicular, o incremento na densidade de plantas dessa cultivar resultou no aumento do comprimento de raízes nas entrelinhas e em profundidade, indicando melhor distribuição horizontal e vertical do sistema radicular. As maiores diferenças entre densidades de plantas foram verificadas nas camadas de $10-20 \mathrm{~cm}$ em ambas as entrelinhas e de 20 30 e 40-50 cm na entrelinha à esquerda, nas quais houve maior comprimento radicular nas maiores densidades (300 ou 450 mil plantas ha-1 $\pm 10 \%$ ). Na linha de semeadura, não houve variação no comprimento radicular entre densidade de plantas, em todas as camadas, inclusive na mais superficial, na qual a menor densidade de plantas resultou em maior massa seca (Figura 2) e área radicular (Figura 4). $\mathrm{Na}$ linha de semeadura e na entrelinha à direita, não houve diferença entre camadas de solo nas três densidades. Na camada superficial das densidades de 150 e 300 mil plantas ha $^{-1} \pm 10 \%$, verificou-se menor comprimento radicular na linha em relação às entrelinhas. Esse comportamento também foi observado na camada de $10-20 \mathrm{~cm}$, na maior densidade de plantas.

Na cultivar BMX Potência RR, a área e o comprimento de raízes foram apenas afetados pelas camadas de solo (Tabela 1). Para as duas variáveis, houve maior crescimento de raízes na camada 
superficial em comparação às camadas subsuperficiais. Ressalta-se que, independentemente da camada e da densidade de plantas, não houve efeito significativo da posição de coleta (linha ou entrelinhas) sobre a área e comprimento radiculares nessa cultivar, evidenciando uniformidade na distribuição horizontal de raízes.

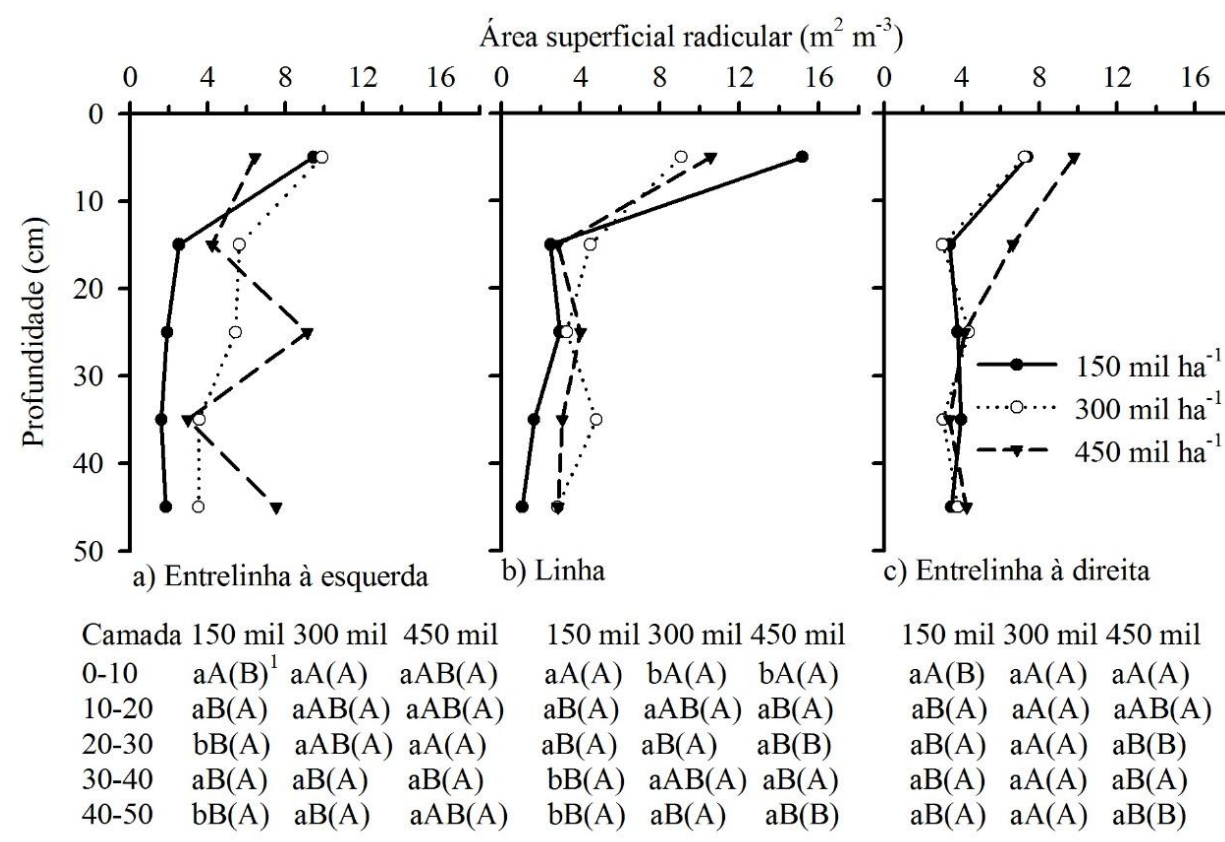

Figura 4. Área superficial radicular, cultivar BRS 359 RR, em diferentes camadas de solo, posições de amostragem e densidades de plantas. Londrina, PR, safra 2014/2015.

1'Letras minúsculas comparam as médias de densidade de plantas dentro de cada posição e camada de solo. Letras maiúsculas comparam as médias de camadas de solo dentro de cada posição e densidade de plantas. Letras entre parênteses comparam as médias de posições de amostragem dentro de cada densidade de plantas e camada de solo (Tukey $\mathrm{p}<0,05)$. CV densidade $=24,2 \%$; CV camada de solo $=27,7 \%$ e CV da posição $=16,7 \%$. Dados transformados em $(x+1)^{1 / 2}$.

Figure 4. Root surface area, cultivar BRS 359 RR, in different soil layers, positions and plant densities. Londrina, PR, 2014/2015 growing season.

${ }^{1}$ Lowercase letters compare averages of plant densities in each position and soil layer. Uppercase letters compare averages of soil layers inside each position and plant densities. Letters in parentheses compare averages of sampling positions inside each plant densities and soil layers (Tukey $p<0.05$ ). CV density $=$ $24.2 \%$; CV soil layer $=27.7 \%$ and $\mathrm{CV}$ position $=16.7 \%$. Transformed data $(x+1)^{1 / 2}$.

O diâmetro médio de raízes ponderado pelo comprimento foi afetado pela interação tripla na cultivar BRS 359 RR (Figura 6). Na camada superficial do solo e na linha de semeadura, houve redução do diâmetro de raízes com o aumento da densidade de plantas. O maior diâmetro radicular explica os maiores valores de densidade de massa seca (Figura 2b) e área superficial (Figura 4b) na camada de 0-10 cm sob a linha de semeadura na densidade de 150 mil plantas ha-1 $\pm 10 \%$, mesmo com esse tratamento apresentando, na mesma posição e camada, um comprimento radicular similar às demais densidades (Figura 5b). Todavia, nas outras camadas e na camada superficial nas entrelinhas, o diâmetro não foi influenciado pelas densidades de plantas. Nas três densidades de plantas, houve maior diâmetro na linha de semeadura do que nas entrelinhas na camada superficial.

Independentemente da camada e da posição horizontal, o diâmetro radicular ponderado pelo comprimento não foi influenciado pelas densidades de plantas na cultivar BMX Potência RR, porém houve interação entre as posições e as camadas amostradas para essa variável (Figura 7). Os resultados do desdobramento dessa interação para a cultivar BMX Potência RR foram similares aos obtidos com a BRS 359 RR. O maior diâmetro de raízes nas camadas de 0-10 e 10-20 cm foi observado na linha de semeadura, mas não houve diferença entre as posições amostradas nas camadas mais profundas. Na linha de semeadura, observou-se redução gradual do diâmetro das raízes com o aumento da profundidade, enquanto que nas entrelinhas não houve diferença no diâmetro das raízes entre as camadas de solo. Esses dados confirmam que as alterações mais evidentes no diâmetro das raízes em função da profundidade ocorrem na linha de semeadura e não nas entrelinhas. 


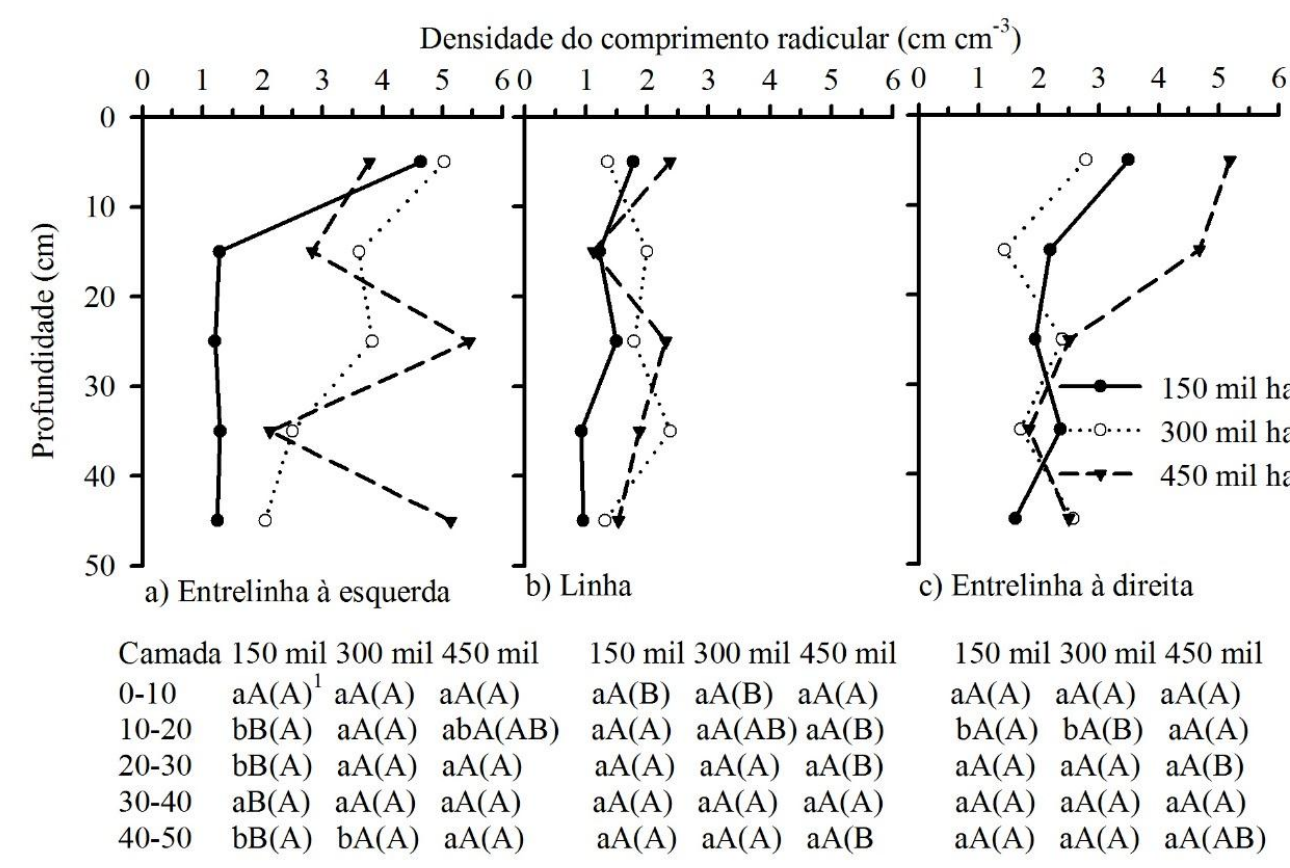

Figura 5. Densidade do comprimento radicular, cultivar BRS 359 RR, em diferentes camadas de solo, posições de amostragem e densidades de plantas. Londrina, PR, safra 2014/2015.

'Letras minúsculas comparam as médias de densidade de plantas dentro de cada posição e camada de solo. Letras maiúsculas comparam as médias de camadas de solo dentro de cada posição e densidade de plantas. Letras entre parênteses comparam as médias de posições dentro de cada densidade de plantas e camada de solo (Tukey p <0,05). CV densidade $=41,8 \%$; CV camada de solo $=34,7 \%$ e CV da posição $=23,3 \%$. Dados transformados em $(x+1)^{1 / 2}$.

Figure 5. Root length density, cultivar BRS 359 RR, in different soil layers, sampling positions and plant densities. Londrina, PR, 2014/2015 growing season.

${ }^{1}$ Lowercase letters compare averages of plants densities in each position and soil layer. Uppercase letters compare averages of soil layers inside each position and plants densities. Letters in parentheses compare averages of sampling positions inside each plant densities and soil layers (Tukey $p<0.05)$. CV density $=$ $41.8 \% ; \mathrm{CV}$ soil layer $=34.7 \%$ and $\mathrm{CV}$ position $=23.3 \%$. Transformed data $(x+1)^{1 / 2}$.

Tabela 1. Área e densidade do comprimento de raízes de soja, cultivar BMX Potência RR, em diferentes camadas de solo. Média de três densidades de plantas e três posições. Londrina, PR, safra $2014 / 2015$.

Table 1. Soybean root area and density of root length, cultivar BMX Potência RR, in different soil layers. Average of three plant densities and three positions. Londrina, PR, 2014/2015 growing season.

\begin{tabular}{ccc}
\hline Camadas de solo $(\mathrm{cm})$ & Área de raízes $\left(\mathrm{m}^{2} \mathrm{~m}^{-3}\right)$ & Comprimento de raízes $\left(\mathrm{cm} \mathrm{cm}^{-3}\right)$ \\
\hline $0-10$ & $8,48 \mathrm{~A}^{1}$ & $2,759 \mathrm{~A}$ \\
$10-20$ & $3,80 \mathrm{~B}$ & $1,887 \mathrm{~B}$ \\
$20-30$ & $3,27 \mathrm{~B}$ & $1,814 \mathrm{~B}$ \\
$30-40$ & $2,70 \mathrm{~B}$ & $1,522 \mathrm{~B}$ \\
$40-50$ & $2,74 \mathrm{~B}$ & $1,632 \mathrm{~B}$ \\
CV $(\%)$ & 19,2 & 22,0 \\
\hline
\end{tabular}

${ }^{1}$ Letras maiúsculas comparam as médias de camadas de solo (Tukey $\left.\mathrm{p}<0,05\right)$. Dados transformados em $(x+1)^{1 / 2}$.

Os resultados obtidos no presente trabalho demonstram claramente que o aumento da densidade de plantas proporcionou melhor distribuição horizontal e vertical do sistema radicular da cultivar BRS 359 RR, com o aumento da quantidade de raízes em camadas subsuperficiais, especialmente nas entrelinhas da cultura. Provavelmente, a melhor distribuição das raízes no perfil do solo em maiores densidades de plantas de soja associa-se ao aumento da competição intraespecífica por água na linha de semeadura e nas camadas superficiais. Essa hipótese é reforçada pela ocorrência de um período relativamente com pouca precipitação que antecedeu a coleta de raízes, início de janeiro (Figura 1), o que provavelmente aumentou a competição por água nas maiores densidades. Adicionalmente, os teores adequados de nutrientes na área, em conjunto com a adubação de acordo com a análise do solo e o requerimento da cultura, evidenciam que a competição por nutrientes não deve ter se constituído em fator determinante da alteração da distribuição 
radicular. Esses resultados corroboram HUTCHINGS \& JOHN (2004), cujos resultados indicam que as plantas podem aumentar a quantidade de raízes produzidas em condições de maior competição intraespecífica.

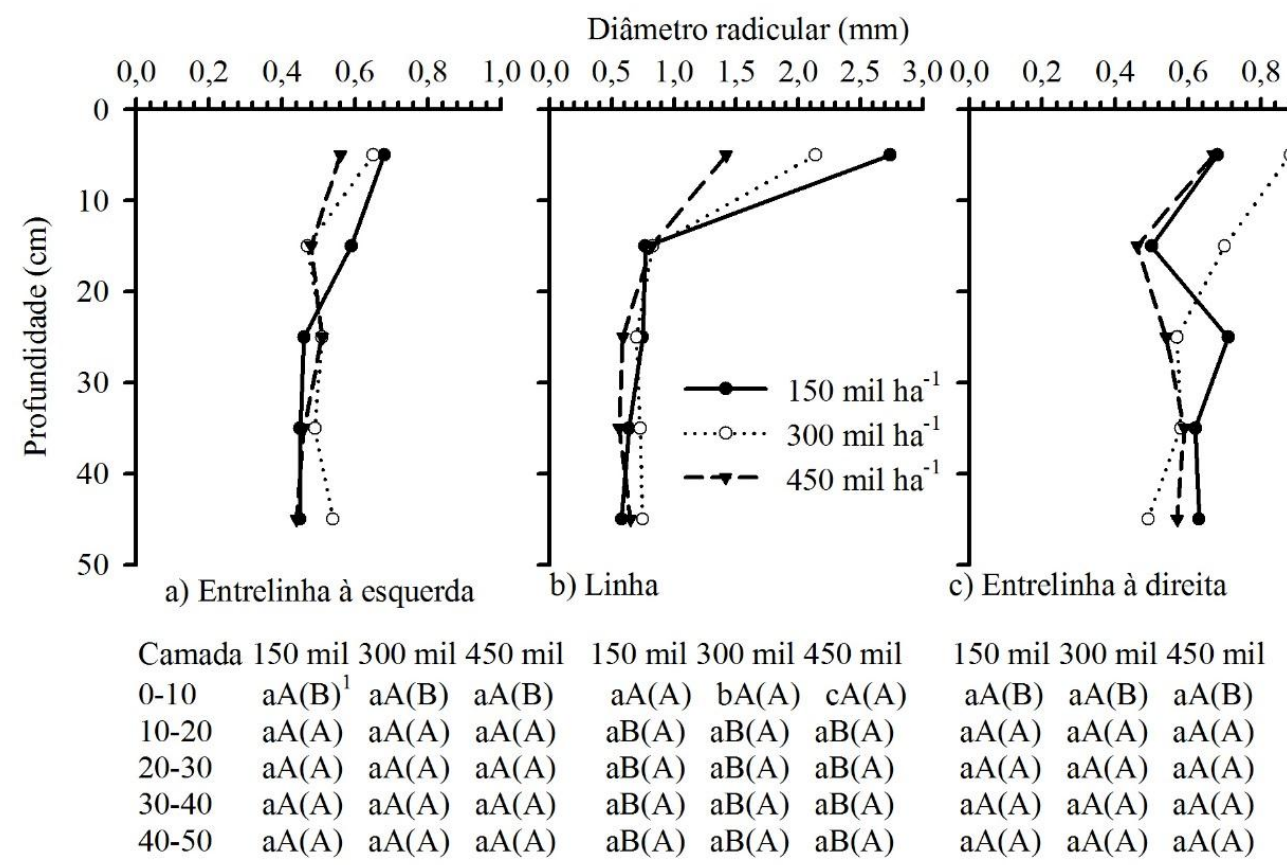

Figura 6. Diâmetro de raízes ponderado pelo comprimento (mm), cultivar BRS 359 RR, em diferentes camadas de solo, posições de amostragem e densidades de plantas. Londrina, PR, safra $2014 / 2015$.

${ }^{1}$ Letras minúsculas comparam as médias de densidade de plantas dentro de cada posição e camada de solo. Letras maiúsculas comparam as médias de camadas de solo dentro de cada posição e densidade de plantas. Letras entre parênteses comparam as médias de posições de amostragem dentro de cada densidade de plantas e camada de solo (Tukey $\mathrm{p}<0,05)$. CV densidade $=47,9 \%$; CV camada de solo $=31,0 \%$ e CV da posição $=32,4 \%$.

Figure 6. Roots diameter weighted by length $(\mathrm{mm})$, cultivar BRS $359 \mathrm{RR}$, in different soil layers, sampling positions and plant densities. Londrina, PR, 2014/2015 growing season.

${ }^{1}$ Lowercase letters compare averages of plant densities in each position and soil layer. Uppercase letters compare averages of soil layers inside each position and plant densities. Letters in parentheses compare averages of sampling positions inside each plant densities and soil layers (Tukey $p<0.05$ ). CV density = $47.9 \%$; CV soil layer $=31.0 \%$ and $\mathrm{CV}$ position $=32.4 \%$.

A melhor distribuição em profundidade das raízes da soja cultivar BRS 359 RR nas maiores densidades de plantas fica ainda mais evidente considerando a proporção da massa, área e comprimento de raízes na camada de $0-10 \mathrm{~cm}$ em relação ao todo $(0-50 \mathrm{~cm})$ (Tabela 2).

Nessa cultivar, a maior concentração de massa, área e comprimento de raízes na camada superficial ocorreu na menor densidade avaliada. No entanto, na cultivar BMX Potência RR, não houve variação significativa entre as densidades para a porcentagem de massa, área e comprimento de raízes na camada superficial do solo. Isso indica que essa cultivar tem um padrão de crescimento e de distribuição de raízes menos afetado pela quantidade de plantas por área.

Os dados da Tabela 2 mostram que, de forma geral para ambas as cultivares, a magnitude da concentração de raízes na camada de $0-10 \mathrm{~cm}$ apresentou dependência em relação à variável considerada, seguindo a ordem: comprimento radicular < área superficial < massa seca. Tal comportamento pode ser atribuído ao maior diâmetro radicular na linha e na camada superficial (Figura 7).

Os efeitos do aumento da competição intraespecífica por recursos ambientais (luz, água e nutrientes) em características morfológicas da parte aérea de plantas de soja têm sido estudados de forma extensiva, sendo relativamente bem conhecidos (BALBINOT JUNIOR et al. 2015a, b). Entretanto, as alterações no crescimento e na distribuição do sistema radicular no perfil do solo em relação ao aumento da competição intraespecífica em maiores densidades de plantas ainda permanecem pouco elucidadas, muito provavelmente pela dificuldade e pelo caráter destrutivo da avaliação das raízes em condições de campo (PANTALONE et al. 1996). 


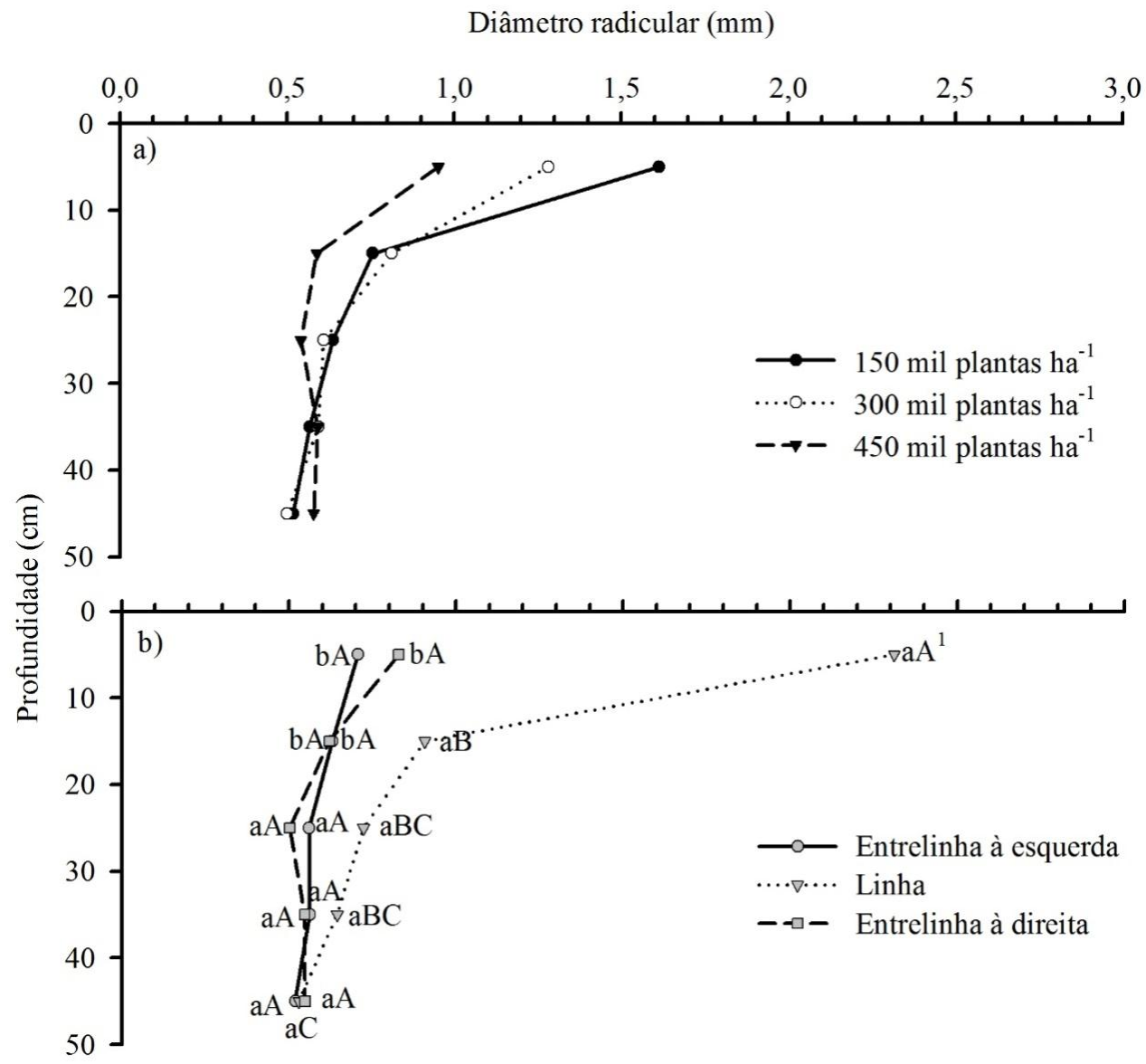

Figura 7. Diâmetro de raízes ponderado pelo comprimento $(\mathrm{mm})$, cultivar BMX Potência RR, em diferentes camadas de solo em função da densidade de plantas (a) e posições de amostragem (b). Londrina, PR, safra 2014/2015.

'Letras minúsculas comparam as médias de posição dentro da camada de solo. Letras maiúsculas comparam as médias de camadas de solo dentro de cada posição (Tukey $p<0,05$ ). CV camada de solo $=$ $30,0 \%$ e CV da posição $=28,0 \%$.

Figure 7. Roots diameter weighted by length $(\mathrm{mm})$, cultivar BMX Potência RR, in different soil layers due on plant densities (a) and sampling positions (b). Londrina, PR, 2014/2015 growing season.

${ }^{1}$ Lowercase letters compare averages of sampling positions inside each soil layers. Uppercase letters compare averages of soil layers inside each sampling position (Tukey $p<0.05$ ). CV soil layer $=30.0 \%$ and $\mathrm{CV}$ position $=28.0 \%$.

Tabela 2. Porcentagem da massa, área e comprimento de raízes de soja presente na camada de 0-10 cm em relação à camada total amostrada $0-50 \mathrm{~cm}$, em duas cultivares e três densidades de plantas. Média de três posições de amostragem. Londrina, PR, safra 2014/2015.

Table 2. Percentage of mass, area and length of soybean roots in the $0-10 \mathrm{~cm}$ layer in relation to the total sampled layer 0-50 cm, at two cultivars and three plant densities. Average of three sampling positions. Londrina, PR, 2014/2015 growing season.

\begin{tabular}{cccc}
\hline $\begin{array}{c}\text { Densidades de } \\
\left.\text { plantas (mil ha }{ }^{-1}\right)\end{array}$ & $\begin{array}{c}\text { Massa seca de raízes } \\
(\%)\end{array}$ & $\begin{array}{c}\text { Área de raízes } \\
(\%)\end{array}$ & $\begin{array}{c}\text { Comprimento de } \\
\text { raízes (\%) }\end{array}$ \\
\hline & & BRS 359 RR & \\
150 & $71,1 \mathrm{~A}^{1}$ & $48,7 \mathrm{~A}$ & $34,0 \mathrm{~A}$ \\
300 & $62,2 \mathrm{AB}$ & $35,7 \mathrm{~B}$ & $24,7 \mathrm{~B}$ \\
CV $(\%)$ & $55,9 \mathrm{~B}$ & $33,4 \mathrm{~B}$ & $24,9 \mathrm{~B}$ \\
& 12,3 & 35,0 & 33,7 \\
150 & & BMX Potência RR & \\
300 & $65,5 \mathrm{~A}$ & $36,0 \mathrm{~A}$ & $21,5 \mathrm{~A}$ \\
450 & $58,4 \mathrm{~A}$ & $40,9 \mathrm{~A}$ & $27,9 \mathrm{~A}$ \\
CV $(\%)$ & $61,3 \mathrm{~A}$ & $38,5 \mathrm{~A}$ & $30,2 \mathrm{~A}$ \\
\hline
\end{tabular}

${ }^{1}$ Letras maiúsculas comparam as médias de densidades de plantas (Tukey $p<0,05$ ). 
De maneira geral, os trabalhos de pesquisa apontam para uma redução (KASPERBAUER 1987, PECHÁČKOVÁ 1999, HÉRBERT et al. 2001, CHENG et al. 2014) ou manutenção (ERICSSON 1995) da quantidade de raízes em função da diminuição da quantidade e/ou qualidade da luz imposta por maiores densidades de plantas. Entretanto, a maior parte desses estudos não tem levado em consideração a distribuição vertical e horizontal do sistema radicular e, principalmente, tem sido conduzida em condições não limitantes de água e nutrientes no solo. Nesse contexto, HUTCHINGS \& JOHN (2004) demonstraram experimentalmente que as plantas podem, em decorrência do aumento da competição, alterar a partição de fotoassimilados entre a parte aérea e as raízes em resposta ao fator ambiental mais limitante ao crescimento (luz, água ou nutrientes). Por exemplo, a maior alocação de fotoassimilados para raízes em detrimento à parte aérea é considerada uma das principais estratégias das plantas para se adaptarem à baixa disponibilidade hídrica no solo (BENJAMIN \& NIELSEN 2006), bem como aumentarem a absorção de nutrientes em condições de baixa fertilidade (ERICSSON 1995, CHENG et al. 2014).

A maior parte dos trabalhos a respeito dos efeitos da competição sobre o crescimento radicular é baseada, principalmente, na análise da massa seca de raízes (KASPERBAUER 1987, ERICSSON 1995, PECHÁČKOVÁ 1999, HÉRBERT et al. 2001, CHENG et al. 2014), mas esse fato pode levar a conclusões incorretas. No presente estudo, a densidade de massa seca radicular se mostrou uma variável menos sensível às alterações na distribuição das raízes no perfil de solo em função da densidade de plantas, comparativamente à área superficial radicular e à densidade do comprimento radicular.

O incremento na quantidade de raízes nas camadas subsuperficiais, principalmente explorando as entrelinhas, pode ser benéfico para períodos com deficiência hídrica, pois a profundidade efetiva do sistema radicular é ampliada, reduzindo a possibilidade de perdas de rendimento em função de estresse hídrico. Portanto, o incremento na profundidade efetiva do sistema radicular, por meio de densidades de plantas adequadas, pode ser um mecanismo-chave na adaptação das plantas a situações de déficit hídrico. Porém, nem sempre o incremento na profundidade radicular está relacionado com incrementos de rendimentos das culturas. Os benefícios de sistemas radiculares mais abundantes e mais bem distribuídos no perfil do solo estão diretamente relacionados com os cenários hídricos de cada cultivo (TARDIEU 2012), sendo mais evidentes em anos secos.

\section{CONCLUSÃO}

O aumento da densidade de plantas provoca a formação de raízes mais finas, com tendência de haver maior ocupação das entrelinhas e das camadas subsuperficiais do solo, mas a magnitude dessa resposta é dependente da cultivar.

\section{REFERÊNCIAS}

BALBINOT JUNIOR AA et al. 2015a. Semeadura cruzada em cultivares de soja com tipo de crescimento determinado. Semina: Ciências Agrárias 36:1215-1226.

BALBINOT JUNIOR AA et al. 2015b. Espaçamento reduzido e plantio cruzado associados a diferentes densidades de plantas em soja. Semina: Ciências Agrárias 36: 2977-2986.

BENJAMIN JG \& NIELSEN DC. 2006. Water deficit effects on root distribution of soybean, field pea and chickpea. Field Crops Research 97: 248-253.

BORDIN I et al. 2008. Matéria seca, carbono e nitrogênio de raízes de soja e milho em plantio direto e convencional. Pesquisa Agropecuária Brasileira 43: 1785-1792.

CHENG L et al. 2014. Interactions between light intensity and phosphorus nutrition affect the phosphate-mining capacity of white lupin (Lupinus albus L.). Journal of Experimental Botany 65: 2995-3003.

COX WJ \& CHERNEY JH. 2011. Growth and yield responses of soybean to row spacing and seeding rate. Agronomy Journal 103:123-128.

ERICSSON T. 1995. Growth and shoot: root ratio of seedlings in relation to nutrient availability. Plant and Soil 168169:205-214.

FAN J et al. 2016. Root distribution by depth for temperate agricultural crops. Field Crops Research 189:68-74.

GASPAR AP \& CONLEY SP. 2015. Responses of canopy reflectance, light interception, and soybean seed yield to replanting suboptimal stands. Crop Science 55: 377-385.

HANNA SO et al. 2008. Fungicide application timing and row spacing effect on soybean canopy penetration and grain yield. Agronomy Journal 100: 1488-1492.

HÉRBERT $Y$ et al. 2001. The response of root/shoot partitioning and root morphology to light reduction in maize genotypes. Crop Science 41: 363-371.

HUTCHINGS MJ \& JOHN EA. 2004. The effects of environmental heterogeneity on root growth and root/shoot partitioning. Annals of Botany 94: 1-8. 
KASPERBAUER MJ. 1987. Far-Red Light reflection from green leaves and effects on phytochrome-mediated assimilate partitioning under field conditions. Plant Physiology 85: 350-354.

PANTALONE VR et al. 1996. Phenotypic evaluation of root traits in soybean and applicability to plant breeding. Crop Science 36: 456-459.

PECHÁČKOVÁ S. 1999. Root response to above-ground light quality - Differences between rhizomatous and nonrhizomatous clones of Festuca rubra. Plant Ecology 141: 67-77.

POSTMA JA et al. 2014. Dynamic root growth and architecture responses to limiting nutrient availability: linking physiological models and experimentation. Biotechnology Advances 32: 53-65.

PROCÓPIO SO et al. 2013. Plantio cruzado na cultura da soja utilizando uma cultivar de hábito de crescimento indeterminado. Revista de Ciências Agrárias/Amazonian Journal of Agricultural and Environmental Sciences 56: 319 325.

TARDIEU F. 2012. Any trait or trait-related allele can confer drought tolerance: just design the right drought scenario. Journal of Experimental Botany 63: 25-31.

WHITE RG \& KIRKEGAARD JA. 2010. The distribution and abundance of wheat roots in a dense, structured subsoil implications for water uptake. Plant, Cell and Environment 33: 133-148. 